\title{
Composite neutrinos and the QCD axion: Baryogenesis, dark matter, small Dirac neutrino masses, and vanishing neutron electric dipole moment
}

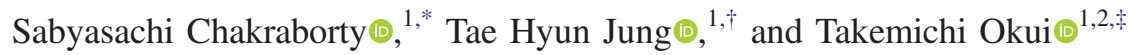 \\ ${ }^{1}$ Department of Physics, Florida State University, Tallahassee, Florida 32306, USA \\ ${ }^{2}$ High Energy Accelerator Research Organization (KEK), Tsukuba 305-0801, Japan
}

(Received 20 August 2021; accepted 24 December 2021; published 21 January 2022)

\begin{abstract}
We consider a chiral gauge theory from which light composite Dirac neutrinos dynamically emerge, augmented by a QCD axion to solve the strong $C P$ problem. We show that an interplay between the composite sector and the axion can also simultaneously lead to successful baryogenesis and generate a correct dark matter abundance via "cogenesis" without contradicting present constraints. We predict $\Delta N_{\text {eff }} \geq 0.14$ and $m_{a}$ in the range $O(10) \mu \mathrm{eV}-O(10) \mathrm{meV}$, which can be firmly tested by upcoming CMB experiments and axion searches.
\end{abstract}

DOI: 10.1103/PhysRevD.105.015024

\section{INTRODUCTION}

Why are neutrinos so light? Why does lepton number appear to be conserved so well? These questions have a beautiful answer for Majorana neutrinos-gauge invariance simply dictates that neutrino masses and associated lepton number violation must originate from an irrelevant interaction suppressed by a large mass scale. But what if neutrinos are Dirac? Do we have a similarly compelling theoretical plot where small neutrino masses and associated lepton number conservation simply emerge from gauge invariance?

Such was provided a long ago by Arkani-Hamed and Grossman [1]. They augmented the SM by a new confining chiral gauge theory whose low energy spectrum below confinement just consists of massless "baryons" $\nu^{\mathrm{c}}$. For example, imagine $\nu^{\mathrm{c}} \sim \Psi \Psi \Psi / \Lambda_{\mathrm{c}}^{3}$ schematically, where $\Psi$ denotes the "quarks" of the new chiral gauge sector and $\Lambda_{c}$ its confinement scale. Then, the leading gauge invariant interaction between $\nu^{\mathrm{C}}$ and SM fields is given by a highly irrelevant operator $H \ell \Psi \Psi \Psi / M^{3}$ suppressed by a high scale $M$. Below $\Lambda_{c}$, this operator becomes a Yukawa interaction $y_{\nu} H \ell \nu^{\mathrm{c}}$ with $y_{\nu} \sim\left(\Lambda_{\mathrm{c}} / M\right)^{3}$, so a mild hierarchy between $\Lambda_{c}$ and $M$ can give us a tiny $y_{\nu}$. This operator accidentally conserves lepton number with $\nu^{\mathrm{c}}$ carrying lepton number -1 . Gauge invariance dictates that the leading

\footnotetext{
*schakraborty5@fsu.edu †thjung0720@gmail.com

tokui@fsu.edu
}

Published by the American Physical Society under the terms of the Creative Commons Attribution 4.0 International license. Further distribution of this work must maintain attribution to the author(s) and the published article's title, journal citation, and DOI. Funded by SCOAP. violation of lepton number conservation comes from $(\Psi \Psi \Psi)^{2} / M^{5} \sim \Lambda_{\mathrm{c}}\left(\Lambda_{\mathrm{c}} / M\right)^{5} \nu^{\mathrm{c}} \nu^{\mathrm{c}}$, an even more irrelevant operator than $H \ell \Psi \Psi \Psi$. Thus, $\nu^{\mathrm{c}}$ and the SM neutrino $\nu$ together acquire a naturally small Dirac mass, with the Majorana mass for $\nu^{\mathrm{c}}$ and associated lepton number violation being automatically negligible. Light Dirac neutrinos and lepton number conservation thus emerge from gauge invariance. ${ }^{1}$

In this paper, we show that just such composite sector for explaining small Dirac neutrino masses augmented by a QCD axion to solve the strong $C P$ problem (i.e., make the neutron EDM vanish) can also simultaneously provide a correct DM abundance and successful baryogenesis, over a large viable parameter range. The first two of these four problems are, of course, solved by construction. However, DM and baryon asymmetry are generated through an intimate interplay between the axion and composite sectors via the "cogenesis" mechanism [3-5], and it is this interplay that permits a large range of parameters in which all four problems are simultaneously solved.

\section{WHAT GOES IN: NEUTRINO MASSES AND STRONG $C P$}

As described above, our theoretical inputs are a composite sector for light Dirac neutrinos and a QCD axion for the strong $C P$ problem. For concreteness we study the following composite sector, but our framework itself is more general and not intrinsically tied to this specific example.

Consider an SU(6) gauge theory with three left-handed Weyl fermions, $\psi_{i}(i=1,2)$ and $\chi$, where $\psi_{i}$ and $\chi$ are in

\footnotetext{
${ }^{1}$ This mechanism has an obvious RS dual [2] if we replace the asymptotically-free gauge dynamics by a strongly coupled CFT.
} 
TABLE I. Symmetries in the composite sector. SU(6) is gauged, while $\mathrm{SU}(2)_{\psi}$ and $\mathrm{U}(1)_{L}$ emerge as accidental global symmetries of the composite sector, both free of anomalies under SU(6). The Weyl fermions $\psi$ and $\chi$ are elementary, while $\nu^{\mathrm{c}}$ is a massless composite "baryon" from SU(6) confinement, carrying the quantum numbers of $\psi \psi \chi \chi$.

\begin{tabular}{lccc}
\hline \hline & \multirow{2}{*}{ Gauge } & \multicolumn{2}{c}{ Global } \\
\cline { 3 - 4 } & $\mathrm{SU}(6)$ & $\mathrm{SU}(2)_{\psi}$ & $\mathrm{U}(1)_{L}$ \\
\hline$\psi$ & $\overline{\mathbf{6}}$ & $\mathbf{2}$ & $-2 / 3$ \\
$\chi$ & $\mathbf{1 5}$ & $\mathbf{1}$ & $1 / 3$ \\
$\nu^{\mathrm{c}} \propto \psi \psi \chi$ & $\mathbf{1}$ & $\mathbf{3}$ & -1 \\
\hline \hline
\end{tabular}

the $\overline{\mathbf{6}}$ and $\mathbf{1 5}$ representations of SU(6), respectively. No masses and interactions with mass dimension $\leq 4$ are allowed by the $\mathrm{SU}(6)$ gauge invariance beside the gauge interaction itself. Consequently, the composite sector Lagrangian accidentally possesses $\mathrm{SU}(2)_{\psi} \times \mathrm{U}(1)_{L}$ global symmetries shown in Table I, which are anomaly free under the $\mathrm{SU}(6)$ gauge interaction and are thus true emergent symmetries of the theory.

Now, what are the light degrees of freedom of the composite sector and how can they interact with SM particles? As discussed in [6], we have compelling arguments, though not a proof, that strongly suggest that this gauge theory undergo confinement without chiral symmetry breaking, where the sole degrees of freedom below the confinement scale are three massless spin-1/2 "baryons" interpolated by the composite operators $\psi_{\{i} \psi_{j\}} \chi$, where $\{i \cdots j\}$ indicates symmetrization of the $\mathrm{SU}(2)_{\psi}$ doublet indices. ${ }^{2}$ The low energy spectrum of the theory, therefore, is given by these three massless Weyl fermions + SM degrees of freedom. The leading gauge-invariant interaction between the massless "baryons" and SM fields appears at dimension-7 and is given by

$$
\frac{1}{M^{3}} H \ell \psi \psi \chi \chi
$$

with some high scale $M$, where $H$ and $\ell$ are the SM Higgs and lepton doublets. Hence, following [1], we identify the three "baryons" with three gauge-singlet fermions for Dirac neutrinos:

$$
\nu^{\mathrm{c}} \sim \frac{(4 \pi)^{2}}{N_{\mathrm{c}} \Lambda_{\mathrm{c}}^{3}} \psi \psi \chi,
$$

\footnotetext{
${ }^{2}$ The conjecture of [6] is that an $\mathrm{SU}(N)$ gauge theory with $N-4$ Weyl fermions in the $\overline{\mathbf{N}}$ representation and one Weyl fermion in the $\mathbf{N}(\mathbf{N}-\mathbf{1}) / \mathbf{2}$ representation should have $(N-3)(N-4) / 2$ massless "baryons" with the $\mathrm{SU}(N-4)$ flavor symmetry unbroken. The $N=6$ case gives exactly $3 \nu^{\mathrm{c}}$ 's. A different conjecture was proposed in [7] that the $\mathrm{SU}(N-4)$ should be spontaneously broken to $\operatorname{Sp}(N-4)(\operatorname{Sp}(N-5))$ for $N=$ even (odd). Amusingly, for our case of $N=6$, the two conjectures agree because $\operatorname{Sp}(2)=$ $\mathrm{SU}(2)$ ! Discrete symmetries are also consistent with our case as analyzed carefully in [8].
}

where $N_{\mathrm{c}}=6$ and we have estimated the coefficient by assuming the 't Hooft coupling, $N_{\mathrm{c}} g_{6}^{2} /(4 \pi)^{2}$, is $O(1)$ at scales around $\Lambda_{\mathrm{c}}$. Tiny Yukawa couplings for Dirac neutrinos (e.g., $y_{\nu} \simeq 3 \times 10^{-13}$ for $m_{\nu} \simeq 0.05 \mathrm{eV}$ ) are then generated below the SU(6) confinement scale $\Lambda_{c}$ as:

$$
\text { operator }(1) \rightarrow y_{\nu} H \ell \nu^{\mathrm{c}}, \quad y_{\nu} \sim \frac{N_{\mathrm{c}}}{(4 \pi)^{2}}\left(\frac{\Lambda_{\mathrm{c}}}{M}\right)^{3} .
$$

Thanks to the third power of $\Lambda_{c} / M$, the extreme smallness of $y_{\nu}$ is reduced to a moderate hierarchy between $\Lambda_{\mathrm{c}}$ and $M$ (roughly $\Lambda_{\mathrm{c}} / M \sim 10^{-4}$ ) [1]. The neutrino masses can have Majorana components coming from the operator $(\psi \psi \chi \chi)^{2}$, but this has dimension 9 so the Majorana components are negligible compared to the Dirac components. The neutrinos thus have to be Dirac [1].

The accidental $\mathrm{U}(1)_{L}$ of the composite sector is identified with the accidental $\mathrm{U}(1)$ lepton number of the SM by the operator (1), hence its name. While $\mathrm{U}(1)_{L}$ is anomaly free under $\mathrm{SU}(6)$, it is now anomalous under $\mathrm{SU}(2)_{W} \times \mathrm{U}(1)_{Y} \cdot \mathrm{U}(1)_{B-L}$ is an accidental, anomaly-free global symmetry of the entire SM + composite theory.

$\mathrm{SU}(2)_{\psi}$ is explicitly broken by charged lepton Yukawa couplings as well as the operator (1) since gauge invariance permits an arbitrary $3 \times 3$ matrix for its coefficients. Nevertheless, thanks to the hierarchy $\Lambda_{\mathrm{c}} \ll M$ and a high dimension of the operator (1), properties of the composite sector, such as the existence of three massless fermions, should not be changed.

Our final ingredient is an axion. To solve the strong $C P$ problem, the Lagrangian must include $a \operatorname{tr}\left[G_{\mu \nu} \tilde{G}^{\mu \nu}\right]$, where $a$ denotes the axion, $G_{\mu \nu}$ the gluon field strength tensor, and $\tilde{G}^{\mu \nu} \equiv \epsilon^{\mu \nu \rho \sigma} G_{\rho \sigma} / 2$ :

$$
\mathcal{L} \supset c_{3} \frac{\alpha_{3}}{4 \pi} \frac{a}{f_{a}} \operatorname{tr}\left[G_{\mu \nu} \tilde{G}^{\mu \nu}\right]
$$

The axion may have more couplings than this but we will see this minimal case with only $c_{3}$ can already provide DM and baryogenesis via its interplay with the composite sector.

\section{WHAT COMES OUT: BARYOGENESIS AND DM, AND MORE}

While our minimal model above structurally ensures that the neutrinos are Dirac and light and that the strong $C P$ problem is solved, the neutrino and axion sectors appear just two independent modules to solve two separate problems. Intriguingly, it turns out that, without any further addition, the two sectors together can also generate correct baryon asymmetry and DM abundance via the "cogenesis" mechanism of [3-5], while still preserving the successes in neutrino masses and strong $C P$. 


\section{A. Baryogenesis}

From a low energy perspective around the electroweak phase transition temperature, our baryogenesis is a leptogenesis in that the baryon asymmetry at the electroweak scale originates from the $B-L$ asymmetry generated at some higher temperature $T_{\mathrm{LD}}$ :

$$
\left.\left.\eta_{B} \equiv \frac{\langle B\rangle}{s}\right|_{T_{\mathrm{EW}}} \simeq \frac{28}{79} \frac{\left\langle B-L_{\mathrm{SM}}\right\rangle}{s}\right|_{T_{\mathrm{LD}}},
$$

where $s$ is the entropy density, $T_{\mathrm{EW}} \sim 130 \mathrm{GeV}$ is the electroweak sphaleron decoupling temperature [9], and $L_{\mathrm{SM}}\left(L_{\mathrm{c}}\right)$ is the contribution from the SM (composite) sector to the total lepton number, $L=L_{\mathrm{SM}}+L_{\mathrm{c}}$.

Unlike the usual leptogenesis from heavy righthanded neutrino decays, however, our nonzero value of $\left.\left\langle B-L_{\mathrm{SM}}\right\rangle\right|_{T_{\mathrm{LD}}}$ is produced via the cogenesis mechanism [3,5], which essentially proceeds as follows (see Appendix A for details). At sufficiently high temperatures where the axion potential is negligible, PQ charge conservation implies

$$
f_{a}^{2} \dot{\theta}+\sum_{P} c_{P} n_{P}=f_{a}^{2} \dot{\theta}_{\mathrm{i}}
$$

where $\theta \equiv a / f_{a}$ with $\equiv \frac{\mathrm{d}}{\mathrm{d} t}$, while $c_{P}$ is the PQ charge of particle species $P$, and $n_{P}$ the number density of $P$ minus that of anti- $P$. By assumption, at some "initial" temperature $T_{\mathrm{i}}$ we have $n_{P}\left(T_{\mathrm{i}}\right)=0$ for all $P$ and a classical axion field configuration with $\dot{\theta}\left(T_{\mathrm{i}}\right)=\dot{\theta}_{\mathrm{i}} \neq 0$ (see [3,4] for a possible origin of $\dot{\theta}_{\mathrm{i}}$ and its implications for the axion quality problem).

Scatterings among particles distribute PQ charge to the $n_{P}$ 's such that the free energy is minimized subject to constraints from all effective conservation laws. In particular, the constraints include $\langle B-L\rangle=0$ with $L=L_{\mathrm{SM}}+L_{\mathrm{c}}$. However, at temperatures below what we call the lepton-number decoupling $(L D)$ temperature $T_{\mathrm{LD}}$, the processes shuffling lepton numbers between $L_{\mathrm{SM}}$ and $L_{\mathrm{c}}$ go out of equilibrium, thus effectively conserving $B-$ $L_{\mathrm{SM}}$ and $L_{\mathrm{c}}$ separately. Minimizing the free energy under all such constraints, we get

$$
\left.\frac{\left\langle B-L_{\mathrm{SM}}\right\rangle}{s}\right|_{\mathrm{LD}} \simeq c_{\mathrm{LD}} \frac{T_{\mathrm{LD}}^{2}}{f_{a}^{2}} Y_{\theta}
$$

where $Y_{\theta} \equiv f_{a}^{2} \dot{\theta} / s$ and $c_{\mathrm{LD}}$ is a linear combination of the $c_{P}$ 's. As shown in the Appendix B, $Y_{\theta}$ is conserved at temperatures well below $f_{a}$ but still sufficiently high that the axion potential is negligible.

Most importantly, $c_{\mathrm{LD}}$ contains $c_{3}$. As shown in the Appendix A, we have $c_{\mathrm{LD}} \simeq-0.04 c_{3}+\cdots$ or $\simeq-0.03 c_{3}+$ $\cdots$ depending on whether electron-Yukawa-mediated processes are in equilibrium or not, respectively, and the ellipses denote $c_{P}$ 's other than $c_{3}$. Hence, even the most minimal axion coupling (4) for the strong $C P$ problem will work also for baryogenesis.

This is a good place to pause and compare our scenario with the minimal cogenesis with just $\mathrm{SM}+$ axion discussed in $[3,5]$. In $[3,5]$, cogenesis directly performs baryogenesis (as opposed to leptogenesis), which gives $\eta_{B} \propto T_{\mathrm{EW}}^{2}$. In contrast, from combining (5) and (7), we have $\eta_{B} \propto T_{\mathrm{LD}}^{2}$, so our baryon asymmetry is enhanced relatively by a factor of $\left(T_{\mathrm{LD}} / T_{\mathrm{EW}}\right)^{2}$. This enhancement allows us to have cogenesis and still solve the strong $C P$ problem by the QCD axion, while in [3,5] the region compatible with cogenesis and current axion bounds turns out to contradict the QCD axion relation $m_{a} f_{a} \sim m_{\pi} f_{\pi}$ (see Refs. $[3,10,11]$ for other proposals to enable cogenesis with the QCD axion).

The estimation of $T_{\mathrm{LD}}$ turns out to be tricky. One might attempt to estimate $T_{\mathrm{LD}}$ as follows. The rate of conversion between $L_{\mathrm{SM}}$ and $L_{\mathrm{c}}$ from the $H \ell \psi \psi \psi \chi$ coupling in (1) is roughly $T^{7} / M^{6}$. Comparing this rate with the expansion rate $\sim T^{2} / M_{\mathrm{Pl}}$, one would then get $T_{\mathrm{LD}} \sim\left(M / M_{\mathrm{Pl}}\right)^{1 / 5} M$. This is naive, however, as it does not take into account $L_{\mathrm{SM}} \leftrightarrow L_{\mathrm{c}}$ shuffling processes due to heavy degrees of freedom responsible for generating (1). Those heavy particles can participate in more efficient 2-to-2 scattering processes and may give a much lower value of $T_{\mathrm{LD}}$ than above. Therefore, $T_{\mathrm{LD}}$ depends on the unknown UV physics and cannot be estimated from the effective operator (1).

As we do not wish to commit to a specific UV model in this work, we regard $T_{\mathrm{LD}}$ as a free parameter hierarchically lower than $M$, i.e., $T_{\mathrm{LD}} \ll M$ (which is satisfied even by the naive estimate). We also expect $T_{\mathrm{LD}} \ll m_{\mathrm{UV}}$, where $m_{\mathrm{UV}}$ denotes the mass scale of the unknown UV particles behind the operator (1). This is because LD is triggered when $T$ drops below $m_{\mathrm{UV}}$ and the number densities of those heavy particles becoming exponentially suppressed. This gives $T_{\mathrm{LD}} \sim m_{\mathrm{UV}} / O(10)$, analogously to the standard estimation of thermal WIMP freeze-out, unless one insists on a really tiny coupling between the SM lepton and heavy particles, defeating the whole point of explaining small neutrino masses by compositeness.

The hierarchy $T_{\mathrm{LD}} \ll m_{\mathrm{UV}}$ allows us to determine $c_{\mathrm{LD}}$ without knowing the unknown UV physics behind the effective coupling (1). Combining (5) and (7), we get

$$
\eta_{B} \simeq \frac{29}{78} c_{\mathrm{LD}} \frac{T_{\mathrm{LD}}^{2}}{f_{a}^{2}} Y_{\theta} .
$$

Note that PQ charge conservation (6) is necessary to obtain (8). Since PQ charge conservation requires neglecting the axion potential, we must demand the axion kinetic energy, $f_{a}^{2} \dot{\theta}^{2} / 2$, to be much larger than the axion potential's height, $\simeq 2 f_{a}^{2} m_{a}^{2}(T)$, where $m_{a}(T)$ takes into account the temperature dependence of the potential. Once the kinetic energy drops below this, the axion field will be trapped. 
As in [4], we define the trapping temperature $T_{\text {trap }}$ via the relation

$$
\dot{\theta}\left(T_{\text {trap }}\right) \equiv 2 m_{a}\left(T_{\text {trap }}\right),
$$

and require $T_{\mathrm{LD}} \gg T_{\text {trap }}$. As shown in the Appendix C, this amounts to a much weaker condition than the requirement that LD should occur before the weak sphaleron turns off, i.e., $T_{\mathrm{LD}} \gg T_{\mathrm{EW}}$.

\section{B. Dark matter}

In cogenesis, $\dot{\theta}$ is also responsible for DM production in addition to baryogenesis [3-5]. This allows the reference to $Y_{\theta}$ in (8) to be removed and gives rise to a relation between $\eta_{B}$ and $\mathrm{DM}$ abundance.

If $3 H\left(T_{\text {trap }}\right)>m_{a}\left(T_{\text {trap }}\right)$, the axion field will slow-roll until the condition $3 H(T)=m_{a}(T)$ is reached at a temperature $T<T_{\text {trap }}$ and then will begin to oscillate. Thus, DM in this case is produced via the standard misalignment mechanism [12-15] with an $O(1)$ misalignment angle. Unlike in the standard case, however, this "initial" misalignment angle is not an input parameter in our scenario and cannot be tuned to a small value to avoid a DM overabundance. Our prediction on $m_{a}$ and $f_{a}$ in this case is represented by the red dot in Fig. 1.

For $3 H\left(T_{\text {trap }}\right)<m_{a}\left(T_{\text {trap }}\right)$, the axion field immediately begins oscillating around the nearest minimum of the potential, thereby becoming cold DM. This is the "axion kinetic misalignment" mechanism of [4]. While the DM number density is given by $s Y_{\theta}$ at $T=T_{\text {trap }}$, the energy density is not given by $m_{a} s Y_{\theta}$ because the axion potential is not quite quadratic for an $O(1)$ angle generically expected

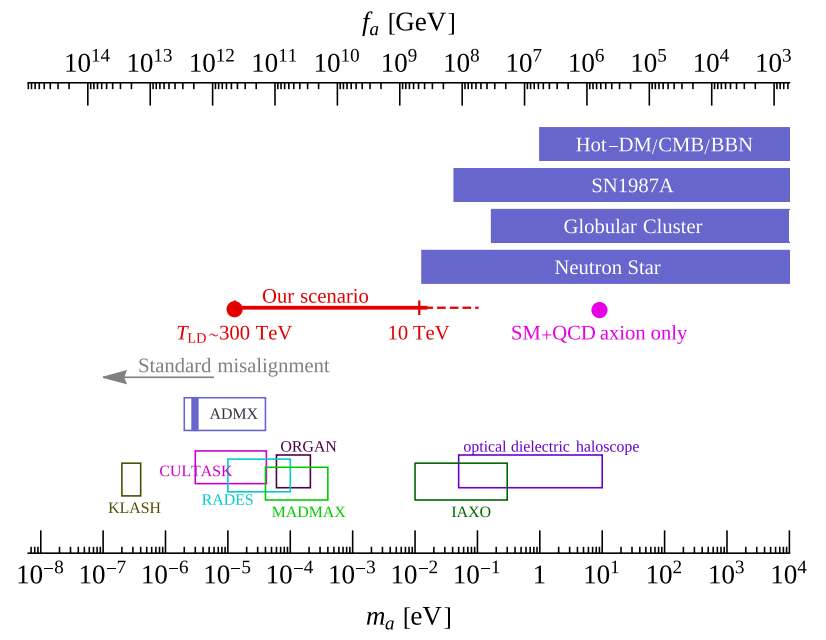

FIG. 1. Our prediction for $c_{\mathrm{LD}}=0.04$ (red line and dot) is depicted along with current constraints (dark blue shaded bands) and sensitivities of future experiments (open rectangles). The magenta dot represents the minimal cogenesis scenario with just $\mathrm{SM}+\mathrm{QCD}$ axion. The gray arrow corresponds to the standard misalignment mechanism for DM. at the moment of trapping. A numerical calculation in [4] shows

$$
\frac{\rho_{\mathrm{DM}}}{s} \simeq 2 m_{a}^{(0)} Y_{\theta}
$$

where $\left.m_{a}^{(0)} \equiv m_{a}(T)\right|_{T=0}$. Using this to eliminate $Y_{\theta}$ in (8), we predict that baryon asymmetry and DM abundance should be related as

$$
\eta_{B} \simeq \frac{29}{78} c_{\mathrm{LD}} \frac{T_{\mathrm{LD}}^{2}}{f_{a} f_{\pi} m_{\pi}} \frac{\rho_{\mathrm{DM}}}{s},
$$

where we have used the leading-order QCD axion relation, $m_{a}^{(0)} f_{a}=z m_{\pi} f_{\pi}$ with $z \equiv \sqrt{m_{u} m_{d}} /\left(m_{u}+m_{d}\right) \simeq 0.5$. In terms of $\Omega_{B} / \Omega_{\mathrm{DM}} \simeq m_{p} \eta_{B} /\left(\rho_{\mathrm{DM}} / s\right)$ with the proton mass $m_{p}$, we thus obtain

$$
\begin{aligned}
\frac{\Omega_{B}}{\Omega_{\mathrm{DM}}} & \simeq \frac{29}{78} c_{\mathrm{LD}} \frac{m_{p} T_{\mathrm{LD}}^{2}}{f_{a} f_{\pi} m_{\pi}} \\
& \simeq 0.2 \frac{c_{\mathrm{LD}}}{0.04}\left(\frac{T_{\mathrm{LD}}}{10 \mathrm{TeV}}\right)^{2} \frac{5 \times 10^{8} \mathrm{GeV}}{f_{a}} .
\end{aligned}
$$

As shown in the Appendix C, the condition $3 H\left(T_{\text {trap }}\right)<$ $m_{a}\left(T_{\text {trap }}\right)$ translates to $T_{\mathrm{LD}} \lesssim 300 \mathrm{TeV}$ for $c_{\mathrm{LD}} \sim 0.04$ and $g_{*}^{\text {trap }} \sim g_{* S}^{\text {trap }} \sim 100$ (and also for $f_{a} \sim 10^{9} \mathrm{GeV}$ but with an extremely mild dependence on $f_{a}$ ). With the lower bound $T_{\mathrm{LD}}>T_{\mathrm{EW}} \sim 0.1 \mathrm{TeV}$, we have a wide possible range of $T_{\mathrm{LD}}$.

In Fig. 1, our prediction (12) with the benchmark value of $c_{\mathrm{LD}}=0.04$ is shown as a red horizontal line segment, where correct baryon asymmetry and DM abundance are generated, the small Dirac neutrino masses are explained, and the strong $C P$ problem is solved. As already mentioned, the red dot corresponds to the case where the standard misalignment is responsible for DM rather than kinetic misalignment. The dark blue shaded bands show constraints from hot $\mathrm{DM} / \mathrm{CMB} / \mathrm{BBN}$ constraints [16], SN1987A [17,18], globular clusters [19], the neutron star cooling rate [20] (which translates to $T_{\mathrm{LD}} \gtrsim$ $10 \mathrm{TeV} \sqrt{0.04 / c_{\mathrm{LD}}}$ ), and ADMX [21]. The magenta dot represents the minimal cogenesis model (i.e., just $\mathrm{SM}+\mathrm{QCD}$ axion) that generates correct baryon asymmetry and DM abundance and solves the strong $C P$ problem, but is excluded by the aforementioned bounds except ADMX. The gray arrow describes $m_{a}$ and $f_{a}$ for the standard misalignment mechanism for DM with an appropriately tuned initial misalignment angle, where the arrow indicates the direction of more tuning. It is an exciting prospect that our parameter range will be probed by a variety of future experiments shown by open rectangles in Fig. 1 [21-29]. 


\section{C. $\Delta N_{\text {eff }}$ and more}

Since we have Dirac neutrinos and our thermal history begins at a temperature above $T_{\mathrm{LD}}$ where the SM particles and the (constituents of) $\nu^{\mathrm{c}}$ 's are in equilibrium, we have a robust prediction on the lower bound on $\Delta N_{\text {eff }}$ :

$$
\Delta N_{\mathrm{eff}} \geq\left.\Delta N_{\mathrm{eff}}\right|_{\min }=3\left(\frac{g_{*}^{\mathrm{SM}}\left(T_{\nu \mathrm{d}}\right)}{g_{*}^{\mathrm{SM}}\left(T_{\mathrm{kd}}\right)}\right)^{4 / 3} \simeq 0.14,
$$

where $T_{\nu \mathrm{d}} \sim O(1) \mathrm{MeV}$ is the neutrino decoupling temperature of the $\mathrm{SM}$, and $T_{\mathrm{kd}}<T_{\mathrm{LD}}$ is the temperature at which the SM and composite sectors kinetically decouple from each other.

The absolute lower bound (13) assumes $T_{\mathrm{kd}}>m_{t}$ to include all SM degrees of freedom in $g_{*}^{\mathrm{SM}}\left(T_{\mathrm{kd}}\right)$ and it is near the edge of the current $1 \sigma$ uncertainty from the Planck experiment [30], $N_{\text {eff }}=2.99 \pm 0.17$. If $T_{\text {kd }}$ falls between the charm mass and QCD confinement temperature, we would have $\Delta N_{\text {eff }} \simeq 0.29$, almost at the $2 \sigma$ bound. Thus, near future $\mathrm{CMB}$ experiments such as the CMB stage-IV and the CORE mission of the ESA [31,32] are expected to offer firm tests of our scenario.

If $T_{\mathrm{kd}}>\Lambda_{\mathrm{c}}$, a large reduction in the degrees of freedom in the composite sector upon confinement would enhance $\Delta N_{\text {eff }}$ by a factor of $\left(g_{*}^{\mathrm{c}+} / g_{*}^{\mathrm{c}-}\right)^{4 / 3}$, where $g_{*}^{\mathrm{c}+(-)}$ is the degrees of freedom in the composite sector above(below) $\Lambda_{\mathrm{c}}$. For the SU(6) benchmark model, the enhancement would give $\Delta N_{\text {eff }} \simeq 8.8$, grossly at odds with observation.

Like $T_{\mathrm{LD}}, T_{\mathrm{kd}}$ depends sensitively on the unknown UV physics behind generating the effective operator (1) so we treat it as a free parameter. All we need to show is that $T_{\mathrm{kd}}$ can be lower than $\Lambda_{\mathrm{c}}$ in some UV models. Generically, UV dynamics generating (1) will also generate interactions of the form $\bar{\ell} \bar{\sigma}^{\mu} \ell \bar{\Psi} \bar{\sigma}_{\mu} \Psi / M^{\prime 2}$ with $\Psi=\psi, \chi$, as these are allowed by all symmetries of the theory. For $T \gtrsim \Lambda_{\mathrm{c}}$, those couplings mediate 2-to-2 processes such as $\ell+\Psi \rightarrow \ell+\Psi$ with a rate of order $\sim T^{5} /\left(4 \pi M^{\prime 4}\right)$. For $T \lesssim \Lambda_{\mathrm{c}}$, they mediate $\ell+\nu^{\mathrm{c}} \rightarrow \ell+\nu^{\mathrm{c}}$, etc., which (accidentally) give rates of the same order, $\sim T^{5} /\left(4 \pi M^{\prime 4}\right)$. Demanding those processes to be in equilibrium, we get $T_{\mathrm{kd}} \sim\left(4 \pi \sqrt{g_{*}} M^{\prime 4} / M_{\mathrm{Pl}}\right)^{1 / 3}$. Then, by using (3), the condition $T_{\mathrm{kd}} \ll \Lambda_{\mathrm{c}}$ gives

$$
\Lambda_{\mathrm{c}} \ll \frac{M_{\mathrm{Pl}}}{4 \pi \sqrt{g_{*}}}\left(\frac{(4 \pi)^{2} y_{\nu}}{N_{\mathrm{c}}}\right)^{\frac{4}{3}}\left(\frac{M}{M^{\prime}}\right)^{4} \sim 10^{2} \mathrm{GeV}\left(\frac{M}{M^{\prime}}\right)^{4}
$$

for $g_{*} \sim 100$ and $y_{\nu} \simeq 3 \times 10^{-13}$. If $M^{\prime}=M$, this would imply $T_{\mathrm{kd}} \ll \Lambda_{\mathrm{c}} \ll 100 \mathrm{GeV}$ so the absolute lower bound (13) cannot be reached because $T_{\mathrm{kd}}$ is below $m_{t}$, although there may barely be room for $T_{\mathrm{kd}}$ to be above the QCD confinement scale to saturate the $2 \sigma$ bound. However, slight differences in the values of UV couplings can easily give $M$ slightly larger than $M^{\prime}$ by, e.g., a factor of 5 , which will open up a wide gap for $T_{\mathrm{kd}}$ to sit between $m_{t}$ and $\Lambda_{\mathrm{c}}$ and thus lead to $\Delta N_{\text {eff }} \simeq 0.14$.
However, the analysis above does suggest our scenario will be testable in near future, not only by CMB but also possibly by laboratory experiments. If we observe $\Delta N_{\text {eff }} \simeq$ 0.14 or some value close to it in future CMB experiments, we should expect a new physics associated with the scale $M^{\prime}$, followed by a new physics associated with $M$ and the operator (1). For example, in some UV models, operators giving rise to $\mu \rightarrow e \gamma$ and/or the electron EDM can be generated along with $\bar{\ell} \bar{\sigma}^{\mu} \ell \bar{\Psi} \bar{\sigma}_{\mu} \Psi / M^{\prime 2}$. The study of such signals is highly UV model dependent by nature, however, and hence beyond the scope of this work.

\section{SUMMARY}

We have shown that the composite origin of small Dirac neutrino masses, when it is combined with a (minimal) QCD axion to solve the strong $C P$ problem, can simultaneously also generate correct baryon asymmetry and DM abundance. Our parameter space is currently wide open with $m_{a}$ in the range between $O(10) \mathrm{meV}$ and $O(10) \mu \mathrm{eV}$, which will be probed in various upcoming experiments. Our scenario also predicts a robust lower bound on $\Delta N_{\text {eff }}$ of 0.14 , which, again, will be unambiguously testable in near future.

\section{ACKNOWLEDGMENTS}

This work is supported by the US Department of Energy Grant No. DE-SC0010102 and the Japan Society for Promotion of Science (JSPS) Grant No. KAKENHI $21 \mathrm{H} 01086$.

\section{APPENDIX A: LEPTON ASYMMETRY}

Here, we summarize the calculations of lepton asymmetry quoted in the main text.

\section{Lagrangian and $P Q$ current}

We start from the basis in which the couplings linear in the axion field $a(x)$ (or $\theta(x) \equiv a(x) / f_{a}$ ) take the following form:

$\mathcal{L}_{a \mathrm{int}}=\frac{\theta}{16 \pi^{2}} \sum_{F} c_{F} g_{F}^{2} \operatorname{tr}\left[F_{\mu \nu} \tilde{F}^{\mu \nu}\right]+\partial_{\mu} \theta \sum_{f} c_{f} \bar{f} \bar{\sigma}^{\mu} f$,

where $F=1,2,3,6$ respectively indicate the $\mathrm{U}(1)_{Y}$, $\mathrm{SU}(2)_{W}, \mathrm{SU}(3)_{\mathrm{c}}, \mathrm{SU}(6)$ gauge groups, while $f=q, u^{\mathrm{c}}, d^{\mathrm{c}}$, $\ell, e^{\mathrm{c}}, \psi, \chi$. There are no other couplings linear in $\theta$ in this basis; for example, such coupling to the Higgs doublet has been set to zero without loss of generality by redefining the SM fermion fields by a hypercharge rotation. We have assumed flavor universality of the axion interactions. So, more explicitly, $c_{q} \bar{q} \sigma^{\mu} q$ should be interpreted as $\sum_{i=1}^{3} c_{q_{i}} \bar{q}_{i} \sigma^{\mu} q_{i}$ with $c_{q_{1}}=c_{q_{2}}=c_{q_{3}} \equiv c_{q}, \quad c_{\psi} \bar{\psi} \sigma^{\mu} \psi$ as $\sum_{i=1}^{2} c_{\psi_{i}} \bar{\psi}_{i} \sigma^{\mu} \psi_{i}$ with $c_{\psi_{1}}=c_{\psi_{2}} \equiv c_{\psi}$, etc. Such flavor universality is automatic in both the KSVZ and DFSZ 
UV constructions unless one chooses to assign flavordependent PQ charges.

To simplify our calculations, we will ignore the up-quark Yukawa coupling, $y_{u}$, in the basis where the quark Yukawa coupling matrices are diagonal. (Here we strictly mean the 1st-generation up quark so $y_{c}$ and $y_{t}$ will not be ignored. For a more precise, but more complicated, formalism with $y_{u} \neq 0$, see [33].) Two things must be mentioned to justify this approximation. First, the existence of a tiny, nontrivial axion potential is quantitatively irrelevant for the baryogenesis process concerned here as it occurs at much higher energy scales than the height of the potential. Hence, making the potential flat by setting $y_{u}=0$ is a good approximation. Second, setting $y_{u}=0$ does not lead to any additional conservation law. Each conservation law would act as a constraint upon the minimization of the free energy to be performed below, so we must make sure that our approximation does not introduce an extra constraint that should not exist. For $y_{u}$, setting it to zero would perturbatively imply conservation of the $u_{1}^{\mathrm{c}}$ number, but the $\mathrm{SU}(3)_{\mathrm{c}}$ sphaleron violates the $u_{1}^{\mathrm{c}}$ number conservation at temperatures relevant for our baryogenesis mechanism. Therefore, we should be able to capture the physics correctly by not requiring the $u_{1}^{\mathrm{c}}$ number conservation despite setting $y_{u}=0$.

This subtlety matters for the electron, however, because it is possible that the processes that violate the $e_{1}^{\mathrm{c}}$ number conservation are not fast enough to be in equilibrium at relevant temperatures. Below we will analyze two cases depending on whether the $e_{1}^{\mathrm{c}}$ number is conserved or not.

Now, with $y_{u}=0$, we will perform a series of field redefinitions to simplify the axion interactions in (A1). First, we redefine $u_{1}^{\mathrm{c}}$ by an appropriate phase to remove the $a G \tilde{G}$ coupling, which amounts to the following replacement of coefficients in (A1):

$c_{3} \rightarrow c_{3}^{\prime}=0, \quad c_{u_{1}^{\mathrm{c}}} \rightarrow c_{u_{1}^{\mathrm{c}}}^{\prime}=c_{u_{1}^{\mathrm{c}}}-c_{3}=c_{u^{\mathrm{c}}}-c_{3}$.

Next, we remove the $a W \tilde{W}$ coupling by performing the $\mathrm{U}(1)_{B}$ rotation:

$$
\begin{aligned}
c_{2} & \rightarrow c_{2}^{\prime}=0, \\
c_{q} & \rightarrow c_{q}^{\prime}=c_{q}-\frac{c_{2}}{9}, \\
c_{u_{1}^{\mathrm{c}}}^{\prime} & \rightarrow c_{u_{1}^{\mathrm{c}}}^{\prime \prime}=c_{u_{1}^{\mathrm{c}}}^{\prime}+\frac{c_{2}}{9}=c_{u^{\mathrm{c}}}-c_{3}+\frac{c_{2}}{9}, \\
c_{u_{2,3}^{\mathrm{c}}} & \rightarrow c_{u_{2,3}^{\mathrm{c}}}^{\prime}=c_{u^{\mathrm{c}}}+\frac{c_{2}}{9}, \\
c_{d^{\mathrm{c}}} & \rightarrow c_{d^{\mathrm{c}}}^{\prime}=c_{d^{\mathrm{c}}}+\frac{c_{2}}{9} .
\end{aligned}
$$

Finally, to rotate away the $a G_{6} \tilde{G}_{6}$ term, we need an accidental global U(1) symmetry that is anomalous under the $\mathrm{SU}(6)$ gauge interaction. Inspecting all the interactions of our entire $\mathrm{SM}+$ composite Lagrangian described in the main text, we see that such U(1) symmetry indeed exists, where $\psi$ and $\chi$ carry charges 1 and -2 , respectively, and no SM fields nor axion are charged. Being anomalous under $\mathrm{SU}(6)$, a rotation in this $\mathrm{U}(1)$ can be used to remove the $a G_{6} \tilde{G}_{6}$ term as

$c_{6} \rightarrow c_{6}^{\prime}=0, \quad c_{\psi} \rightarrow c_{\psi}^{\prime}=c_{\psi}+\frac{c_{6}}{6}, \quad c_{\chi} \rightarrow c_{\chi}^{\prime}=c_{\chi}-\frac{c_{6}}{3}$.

We do not have an anomalous global symmetry we could use to remove the $a B \tilde{B}$ term. Nevertheless, since $\int \mathrm{d}^{4} x B \tilde{B}$ is a vanishing surface integral, the presence of $a B \tilde{B}$ will have no effects on our baryogenesis mechanism. If the $e_{1}^{\mathrm{c}}$ number violating interactions are too slow to be in equilibrium, we can set $y_{e}=0$ and this will in turn allow us to rotate away $a B \tilde{B}$ by redefining $e_{1}^{\mathrm{c}}$. This might appear to contradict the statement that $B \tilde{B}$ is irrelevant, because $c_{1}$ will still be in the Lagrangian as part of $c_{e_{1}^{\mathrm{c}}}^{\prime}$, just as $c_{3}$ is part of $c_{u_{1}^{\mathrm{c}}}^{\prime}$. But precisely because of the $e_{1}^{\mathrm{c}}$ conservation that needs to be imposed in this case, $c_{e_{1}^{\mathrm{c}}}^{\prime}$ will drop out from our calculation and hence the contradiction will not arise.

Our Lagrangian is now manifestly invariant under a global shift of the axion field, $\theta(x) \rightarrow \theta(x)+\alpha$ with a spacetime constant $\alpha$, except for the $a B \tilde{B}$ term. This implies the conservation law:

$$
\partial_{\mu} J_{\mathrm{PQ}}^{\mu}=c_{1}^{\prime} \frac{g_{1}^{2}}{16 \pi^{2}} B_{\mu \nu} \tilde{B}^{\mu \nu}
$$

with

$J_{\mathrm{PQ}}^{\mu}=f_{a}^{2} \partial^{\mu} \theta+\sum_{f} c_{f} \bar{f} \bar{\sigma}^{\mu} f$ with replacements (A2)-(A8).

We do not need an expression for $c_{1}^{\prime}$ as the right-hand side of (A9) will vanish when integrated over spacetime, implying that the total PQ charge, $\int \mathrm{d}^{3} x J_{\mathrm{PQ}}^{0}$, is conserved regardless of the value of $c_{1}^{\prime}$. If we had chosen not to neglect $y_{u}$, a term of the form i $y_{u} H q u^{\mathrm{c}}+$ c.c. would appear on the right-hand side above and $J_{\mathrm{PQ}}^{\mu}$ would no longer be conserved.

We assume that the fermions' and scalars' number densities as well as the axion field are spatially homogeneous throughout the baryogenesis process, so we always have $J_{\mathrm{PQ}}^{1,2,3}=0$. We also assume vanishing initial number densities for all the $f$ 's and $\Phi$ 's, so the initial condition is given by $J_{\mathrm{PQ}}^{0}=f_{a}^{2} \dot{\theta}_{\mathrm{i}}$. Then, the PQ charge conservation implies that at later times we have 


$$
R_{\mathrm{i}}^{3} f_{a}^{2} \dot{\theta}_{\mathrm{i}}=R^{3}\left(f_{a}^{2} \dot{\theta}+\sum_{f} c_{f} n_{f}\right)
$$

with replacements (A2)-(A8),

where $R\left(R_{\mathrm{i}}\right)$ is the (initial) scale factor of the expanding universe, and $n_{f} \equiv \bar{f} \bar{\sigma}^{0} f$. Assuming entropy conservation $s_{\mathrm{i}} R_{\mathrm{i}}^{3}=s R^{3}$, this becomes

$$
\frac{f_{a}^{2} \dot{\theta}_{\mathrm{i}}}{s_{\mathrm{i}}}=\frac{f_{a}^{2} \dot{\theta}+n_{\mathrm{PQ}}}{s},
$$

with

$$
\begin{aligned}
n_{\mathrm{PQ}} \equiv & \left(c_{u^{\mathrm{c}}}+\frac{c_{2}}{9}-c_{3}\right) n_{u_{1}^{\mathrm{c}}}+\left(c_{u^{\mathrm{c}}}+\frac{c_{2}}{9}\right) n_{u_{23}^{\mathrm{c}}} \\
& +\left(c_{d^{\mathrm{c}}}+\frac{c_{2}}{9}\right) n_{d^{\mathrm{c}}}+\left(c_{q}-\frac{c_{2}}{9}\right) n_{q}+c_{\ell} n_{\ell}+c_{e^{\mathrm{c}}} n_{e^{\mathrm{c}}} \\
& +\left(c_{\psi}+\frac{c_{6}}{6}\right) n_{\psi}+\left(c_{\chi}-\frac{c_{6}}{3}\right) n_{\chi},
\end{aligned}
$$

where

$$
\begin{aligned}
n_{f} & \equiv n_{f_{1}}+n_{f_{2}}+n_{f_{3}} \quad \text { for } f=q, d^{\mathrm{c}}, \ell, e^{\mathrm{c}}, \\
n_{u_{23}^{\mathrm{c}}} & \equiv n_{u_{2}^{\mathrm{c}}}+n_{u_{3}^{\mathrm{c}}}, \quad n_{\psi} \equiv n_{\psi_{1}}+n_{\psi_{2}} .
\end{aligned}
$$

\section{Minimizing the free energy and determining the asymmetries}

We will determine the values of the number densities in (A12) by minimizing the free energy. We write the free energy density $\mathcal{F}$ as

$$
\mathcal{F}=\rho_{a}+\rho_{\mathrm{p}}-T s_{\mathrm{p}},
$$

where $\rho_{a}=f_{a}^{2} \dot{\theta}^{2} / 2$ is the energy density of the classical motion of the axion field, while $\rho_{\mathrm{p}}$ and $s_{\mathrm{p}}$ are respectively the energy and entropy densities of the plasma. Using (A12) and (A13), we can express $\rho_{a}$ in terms of the number densities as

$$
\rho_{a}=\frac{1}{2 f_{a}^{2}}\left(\frac{s}{s_{\mathrm{i}}} f_{a}^{2} \dot{\theta}_{\mathrm{i}}-n_{\mathrm{PQ}}\right)^{2} .
$$

We will also express $\rho_{\mathrm{p}}$ and $s_{\mathrm{p}}$ in terms of the number densities below. Then, $\mathcal{F}$ will be a function of the number densities, and the values of the number densities at equilibrium should minimize $\mathcal{F}$. The minimization determines the number densities completely in terms of the $c$ coefficients and $\dot{\theta}$ without needing to solve a large number of Boltzmann equations as in Refs. [3,33]. This simplicity is made possible because of the PQ charge conservation (A12) coming from our approximation of setting $y_{u}=0$. For $y_{u} \neq 0$, the Boltzmann equations would be needed.
To calculate $\rho_{\mathrm{p}}$ and $s_{\mathrm{p}}$ in terms of the number densities, let us first work out relations between the number density, $n_{i}$, and chemical potential, $\mu_{i}$, of a particle species $i$. Neglecting interactions between particles, for a free massless fermion $f$ with degeneracy $N_{f}$ in equilibrium at $T \gg \mu_{f}$, we have

$$
\begin{aligned}
n_{f} & =N_{f} \int \frac{\mathrm{d}^{3} p}{(2 \pi)^{3}} \frac{1}{e^{\left(E-\mu_{f}\right) / T}+1}-\left(\mu_{f} \rightarrow-\mu_{f}\right) \\
& =\frac{N_{f}}{6} \mu_{f} T^{2}+O\left(\mu_{f}^{3}\right) .
\end{aligned}
$$

Similarly, for a free massless scalar boson $b$ with degeneracy $N_{b}$, we have

$$
\begin{aligned}
n_{b} & =N_{b} \int \frac{\mathrm{d}^{3} p}{(2 \pi)^{3}} \frac{1}{e^{\left(E-\mu_{b}\right) / T}-1}-\left(\mu_{b} \rightarrow-\mu_{b}\right) \\
& =\frac{N_{b}}{3} \mu_{b} T^{2}+O\left(\mu_{b}^{3}\right) .
\end{aligned}
$$

Therefore, neglecting the $O\left(\mu_{f, b}^{3}\right)$ corrections, we have

$$
\mu_{f}=\frac{6 n_{f}}{N_{f} T^{2}}, \quad \mu_{b}=\frac{3 n_{b}}{N_{b} T^{2}} .
$$

Using these, we can express the energy densities of $f$ and $b$ in terms of $n_{f}$ and $n_{b}$ as

$$
\begin{aligned}
\rho_{f} & =N_{f} \int \frac{\mathrm{d}^{3} p}{(2 \pi)^{3}} \frac{E}{e^{\left(E-\mu_{f}\right) / T}+1}+\left(\mu_{f} \rightarrow-\mu_{f}\right) \\
& =N_{f}\left(\frac{7}{8} \frac{\pi^{2}}{15} T^{4}+\frac{1}{4} \mu_{f}^{2} T^{2}\right)+O\left(\mu_{f}^{4}\right) \\
& =\frac{\pi^{2}}{15} \frac{7 N_{f}}{8} T^{4}+9 \frac{n_{f}^{2}}{N_{f} T^{2}}+O\left(n_{f}^{4}\right), \\
\rho_{b} & =N_{b} \int \frac{\mathrm{d}^{3} p}{(2 \pi)^{3}} \frac{E}{e^{\left(E-\mu_{f}\right) / T}-1}+\left(\mu_{b} \rightarrow-\mu_{b}\right) \\
& =N_{b}\left(\frac{\pi^{2}}{15} T^{4}+\frac{1}{2} \mu_{f}^{2} T^{2}\right)+O\left(\mu_{b}^{4}\right) \\
& =\frac{\pi^{2}}{15} N_{b} T^{4}+\frac{9}{2} \frac{n_{b}^{2}}{N_{b} T^{2}}+O\left(n_{b}^{4}\right) .
\end{aligned}
$$

Thus, up to $O\left(n_{f, b}^{4}\right)$ corrections, the total energy density of the plasma becomes

$$
\rho_{\mathrm{p}}=\frac{\pi^{2}}{30} g_{*} T^{4}+9 \sum_{f} \frac{n_{f}^{2}}{N_{f} T^{2}}+\frac{9}{2} \sum_{b} \frac{n_{b}^{2}}{N_{b} T^{2}}
$$

where 


$$
\begin{aligned}
f= & q, u_{1}^{\mathrm{c}}, u_{23}^{\mathrm{c}}, d^{\mathrm{c}}, \ell, e^{\mathrm{c}}, \psi, \chi \\
& \quad \text { with } \quad\left(N_{q}, N_{u_{1}^{\mathrm{c}}}, N_{u_{23}^{\mathrm{c}}}, N_{d^{\mathrm{c}}}, N_{\ell}, N_{e^{\mathrm{c}}}, N_{\psi}, N_{\chi}\right) \\
= & (18,3,6,9,6,3,12,15), \\
b= & H \quad \text { with } \quad N_{H}=2 .
\end{aligned}
$$

(If the $e_{1}^{\mathrm{c}}$ number violating processes are out of equilibrium, we would need to separate out $e_{1}^{\mathrm{c}}$ from $e_{2,3}^{\mathrm{c}}$ set $n_{e_{1}^{\mathrm{c}}}=0$, but this can be effectively achieved without altering the notations by simply changing $N_{e^{\text {c }}}$ from 3 to 2 .) Therefore, from $\rho-T s+P=\sum n \mu$ with $P=\rho / 3$ for free massless particles, we have

$$
\begin{aligned}
\rho_{\mathrm{p}}-T s_{\mathrm{p}} & =-\frac{\rho_{\mathrm{p}}}{3}+\sum_{i=f, b} n_{i} \mu_{i} \\
& =-\frac{\pi^{2}}{90} g_{*} T^{4}+3 \sum_{f} \frac{n_{f}^{2}}{N_{f} T^{2}}+\frac{3}{2} \sum_{b} \frac{n_{b}^{2}}{N_{b} T^{2}} .
\end{aligned}
$$

Combining this with (A16) as in (A15), we finally obtain

$$
\begin{aligned}
\mathcal{F}= & \frac{1}{2 f_{a}^{2}}\left(\frac{s}{s_{\mathrm{i}}} f_{a}^{2} \dot{\theta}_{\mathrm{i}}-n_{\mathrm{PQ}}\right)^{2}+3 \sum_{f} \frac{n_{f}^{2}}{N_{f} T^{2}} \\
& +\frac{3}{2} \sum_{b} \frac{n_{b}^{2}}{N_{b} T^{2}}-\frac{\pi^{2}}{90} g_{*} T^{4},
\end{aligned}
$$

where $f, b$ are given in (A23).

Now, the minimization of $\mathcal{F}$ in (A25) is subject to constraints due to conservation laws. For example, the total hypercharge must be zero, $\langle Y\rangle=0$, at all temperatures. There can be other conserved quantities depending on $T$. The conservation/violation of the $e_{1}^{\mathrm{c}}$ number must be also taken into account. We consider each case separately below:

(i) $T_{\mathrm{LD}} \lesssim T \ll m_{\mathrm{UV}}, e_{1}^{\mathrm{c}}$ number not conserved: In this case, the only conserved quantities are $Y$ and $B-L$ as the processes converting between $L_{\mathrm{SM}}$ and $L_{\mathrm{c}}$ are still in equilibrium. So, we minimize $\mathcal{F}$ with respect to the number densities subject to these two constraints:

$$
\begin{aligned}
0= & \langle Y\rangle \\
= & \frac{1}{6} n_{q}-\frac{2}{3}\left(n_{u_{1}^{\mathrm{c}}}+n_{u_{23}^{\mathrm{c}}}\right)+\frac{1}{3} n_{d^{\mathrm{c}}}-\frac{1}{2} n_{\ell}+n_{e^{\mathrm{c}}}+\frac{1}{2} n_{H}, \\
0= & \langle B-L\rangle \\
= & \frac{1}{3}\left(n_{q}-n_{u_{1}^{\mathrm{c}}}-n_{u_{23}^{\mathrm{c}}}-n_{d^{\mathrm{c}}}\right)-\left(n_{\ell}-n_{e^{\mathrm{c}}}\right) \\
& -\left(-\frac{2}{3} n_{\psi}+\frac{1}{3} n_{\chi}\right) .
\end{aligned}
$$

For $T \ll f_{a}$, the results are

$$
\begin{aligned}
\frac{\langle B\rangle}{s}= & \frac{\langle L\rangle}{s} \\
= & \left(\frac{14}{117} c_{3}-\frac{62}{351} c_{2}-\frac{7}{78} c_{6}+\frac{31}{39} c_{q}-\frac{14}{39} c_{u^{\mathrm{c}}}\right. \\
& -\frac{17}{39} c_{d^{\mathrm{c}}}+\frac{8}{39} c_{\ell}-\frac{3}{26} c_{e^{\mathrm{c}}} \\
& \left.-\frac{28}{117} c_{\psi}+\frac{35}{234} c_{\chi}\right) \frac{\dot{\theta}_{\mathrm{i}}}{s_{\mathrm{i}}} T^{2},
\end{aligned}
$$

and

$$
\begin{aligned}
\frac{\left\langle B-L_{\mathrm{SM}}\right\rangle}{s}= & \frac{\left\langle L_{\mathrm{c}}\right\rangle}{s} \\
= & \left(-\frac{35}{936} c_{3}-\frac{49}{702} c_{2}-\frac{79}{312} c_{6}+\frac{49}{156} c_{q}\right. \\
& +\frac{35}{312} c_{u^{\mathrm{c}}}-\frac{133}{312} c_{d^{\mathrm{c}}}-\frac{49}{156} c_{\ell}+\frac{7}{104} c_{e^{\mathrm{c}}} \\
& \left.-\frac{79}{117} c_{\psi}+\frac{395}{936} c_{\chi}\right) \frac{\dot{\theta}_{\mathrm{i}}}{s_{\mathrm{i}}} T^{2} .
\end{aligned}
$$

(i') $T_{\mathrm{LD}} \lesssim T \ll m_{\mathrm{UV}}, e_{1}^{\mathrm{c}}$ number conserved: As explained earlier, this case can be obtained by redoing case (i) with $N_{e^{\mathrm{c}}}=2$ instead of 3 . The results for $T \ll f_{a}$ are:

$$
\begin{aligned}
\frac{\langle B\rangle}{s}= & \frac{\langle L\rangle}{s} \\
= & \left(\frac{95}{846} c_{3}-\frac{220}{1269} c_{2}-\frac{13}{141} c_{6}+\frac{110}{141} c_{q}\right. \\
& -\frac{95}{282} c_{u^{\mathrm{c}}}-\frac{125}{282} c_{d^{\mathrm{c}}}+\frac{31}{141} c_{\ell}-\frac{4}{47} c_{e^{\mathrm{c}}} \\
& \left.-\frac{104}{423} c_{\psi}+\frac{65}{423} c_{\chi}\right) \frac{\dot{\theta}_{\mathrm{i}}}{s_{\mathrm{i}}} T^{2},
\end{aligned}
$$

and

$$
\begin{aligned}
\frac{\left\langle B-L_{\mathrm{SM}}\right\rangle}{s}= & \frac{\left\langle L_{\mathrm{c}}\right\rangle}{s} \\
= & \left(-\frac{14}{423} c_{3}-\frac{91}{1269} c_{2}-\frac{71}{282} c_{6}+\frac{91}{282} c_{q}\right. \\
& +\frac{14}{141} c_{u^{\mathrm{c}}}-\frac{119}{282} c_{d^{\mathrm{c}}}-\frac{91}{282} c_{\ell}+\frac{7}{141} c_{e^{\mathrm{c}}} \\
& \left.-\frac{284}{423} c_{\psi}+\frac{355}{846} c_{\chi}\right) \frac{\dot{\theta}_{\mathrm{i}}}{s_{\mathrm{i}}} T^{2} .
\end{aligned}
$$

(ii) $T \ll T_{\mathrm{LD}}, e_{1}^{\mathrm{c}}$ number not conserved: At these temperatures, the processes converting between $L_{\mathrm{SM}}$ and $L_{\mathrm{c}}$ have frozen out. Therefore, we now have three constraints to be imposed upon minimizing $\mathcal{F}$ : 


$$
\langle Y\rangle=0, \quad \frac{\left\langle B-L_{\mathrm{SM}}\right\rangle}{s}=\left.\frac{\left\langle B-L_{\mathrm{SM}}\right\rangle}{s}\right|_{\mathrm{LD}},
$$

$\frac{\left\langle L_{\mathrm{c}}\right\rangle}{s}=\left.\frac{\left\langle B-L_{\mathrm{SM}}\right\rangle}{s}\right|_{\mathrm{LD}}$,

where $\left\langle B-L_{\mathrm{SM}}\right\rangle_{\mathrm{LD}}$ is the frozen-out value of $\left\langle B-L_{\mathrm{SM}}\right\rangle$ at the lepton number decoupling. Minimizing $\mathcal{F}$ under these constraints, we obtain

$$
\begin{aligned}
\frac{\langle B\rangle}{s}= & \left.\frac{28}{79} \frac{\left\langle B-L_{\mathrm{SM}}\right\rangle}{s}\right|_{\mathrm{LD}}+\left(\frac{21}{158} c_{3}-\frac{12}{79} c_{2}+\frac{54}{79} c_{q}\right. \\
& \left.-\frac{63}{158} c_{u^{\mathrm{c}}}-\frac{45}{158} c_{d^{\mathrm{c}}}+\frac{25}{79} c_{\ell}-\frac{11}{79} c_{e^{\mathrm{c}}}\right) \frac{\dot{\theta}_{\mathrm{i}}}{s_{\mathrm{i}}} T^{2} .
\end{aligned}
$$

Evaluating this expression at $T=T_{\mathrm{EW}}$ at which the $\mathrm{SU}(2)_{W}$ sphalerons freeze out provides us with the final value of $\langle B\rangle$. We ignore the second term above as its $T^{2}$ scaling implies that it is negligible compared to the first term.

(ii') $T \ll T_{\mathrm{LD}}, e_{1}^{\mathrm{c}}$ number conserved: There is no need to analyze this case, because at temperatures near, but still above, the electroweak scale, the $e_{1}^{\mathrm{c}}$ number violating interactions mediated by $y_{e}$ are in equilibrium.

\section{APPENDIX B: CONSERVATION OF $Y_{\theta}$}

The results above tell us that

$$
\frac{n_{\mathrm{PQ}}}{s} \sim T^{2} \frac{\dot{\theta}_{\mathrm{i}}}{s_{\mathrm{i}}} \ll f_{a}^{2} \frac{\dot{\theta}_{\mathrm{i}}}{s_{\mathrm{i}}}
$$

for $T \ll f_{a}$. Then, since we are always in this temperature regime, the $n_{\mathrm{PQ}}$ term in the $\mathrm{PQ}$ charge conservation relation (A12) is negligible and hence $Y_{\theta}$ is conserved to an excellent approximation. This is valid under the condition $T \gg T_{\text {trap }}$ so that the axion potential can be ignored to justify PQ charge conservation (A12) in the first place.

\section{APPENDIX C: TRAPPING TEMPERATURE}

Here, we estimate $T_{\text {trap }}$ defined in (9) and analyze what the conditions $T_{\mathrm{LD}} \gg T_{\text {trap }}$ and $3 H\left(T_{\text {trap }}\right) \ll m_{a}\left(T_{\text {trap }}\right)$ imply.

First, we want to relate $\dot{\theta}\left(T_{\text {trap }}\right)$ in (9) to the observed baryon asymmetry, $\eta_{B} \simeq 8.5 \times 10^{-11}$. Using the conservation of $Y_{\theta}$, which we expect to be still roughly valid at $T_{\text {trap }}$, we obtain $\dot{\theta}\left(T_{\text {trap }}\right)$ from the relation (8):

$$
\dot{\theta}\left(T_{\text {trap }}\right) \simeq \frac{78}{29} \frac{2 \pi^{2}}{45} \frac{\eta_{B} g_{* S}^{\text {trap }}}{c_{\mathrm{LD}}} \frac{T_{\text {trap }}^{3}}{T_{\mathrm{LD}}^{2}}
$$

Next, we want to estimate $m_{a}\left(T_{\text {trap }}\right)$ in (9). Since our axion is solving the strong $C P$ problem, the axion potential $\sim f_{a}^{2} m_{a}^{2}(T)$ is generated from QCD. A recent lattice calculation [15] has shown that the potential scales as $\left(T_{\mathrm{QCD}} / T\right)^{2 n}$ for $T>T_{\mathrm{QCD}}$ with $T_{\mathrm{QCD}} \simeq 150 \mathrm{MeV}$ and $n \simeq 4$. Combining all these, (9) gives

$$
\begin{aligned}
T_{\text {trap }} & \sim\left(\frac{2 \cdot 29}{78} \frac{45}{2 \pi^{2}} \frac{c_{\mathrm{LD}}}{\eta_{B} g_{* S}^{\text {trap }}} m_{a}^{(0)} T_{\mathrm{QCD}}^{n} T_{\mathrm{LD}}^{2}\right)^{\frac{1}{n+3}} \\
& \sim\left(\frac{c_{\mathrm{LD}}}{\eta_{B} g_{* S}^{\text {trap }}} \frac{T_{\mathrm{QCD}}^{n+2} T_{\mathrm{LD}}^{2}}{f_{a}}\right)^{\frac{1}{n+3}}
\end{aligned}
$$

where we have used $m_{a}^{(0)} \simeq z m_{\pi} f_{\pi} / f_{a} \simeq 0.25 T_{\mathrm{QCD}}^{2} / f_{a}$ and dropped the factor of $\left(0.25 \frac{2.29}{78} \frac{45}{2 \pi^{2}}\right)^{1 /(n+3)} \simeq 1$ in the last step. With this, the condition $T_{\text {trap }} \gg T_{\mathrm{QCD}}$ to justify the use of the scaling $m_{a}(T) \propto T^{-n}$ is equivalent to

$T_{\mathrm{LD}} \gg \sqrt{\frac{\eta_{B} g_{* S}^{\text {trap }}}{c_{\mathrm{LD}}} T_{\mathrm{QCD}} f_{a}} \sim 10 \mathrm{GeV} \sqrt{\frac{g_{* S}^{\text {trap }}}{100} \frac{0.04}{c_{\mathrm{LD}}} \frac{f_{a}}{10^{9} \mathrm{GeV}}}$.

This condition is always satisfied because it is weaker than the condition that lepton-number decoupling should occur before the weak sphaleron turns off, i.e., $T_{\mathrm{LD}} \gg$ $T_{\mathrm{EW}} \sim 100 \mathrm{GeV}$.

To justify our use of PQ charge conservation during leptogenesis, we must require $T_{\mathrm{LD}} \gg T_{\text {trap }}$. With (C2), this is equivalent to imposing that

$$
\begin{aligned}
T_{\mathrm{LD}} & \gg\left(\frac{c_{\mathrm{LD}}}{\eta_{B} g_{* S}^{\text {trap }}} \frac{T_{\mathrm{QCD}}}{f_{a}}\right)^{\frac{1}{n+1}} T_{\mathrm{QCD}} \\
& \sim 40 \mathrm{MeV}\left(\frac{c_{\mathrm{LD}}}{0.04} \frac{100}{g_{* S}^{\text {trap }}} \frac{10^{9} \mathrm{GeV}}{f_{a}}\right)^{\frac{1}{5}},
\end{aligned}
$$

which, again, is already satisfied.

Next, we need to require $3 H\left(T_{\text {trap }}\right) \ll m_{a}\left(T_{\text {trap }}\right)$ as explained the main text. For a radiation dominated universe, this condition means

$$
\frac{\sqrt{g_{*}^{\text {trap }}} T_{\text {trap }}^{2}}{M_{\mathrm{Pl}}} \ll m_{a}^{(0)}\left(\frac{T_{\mathrm{QCD}}}{T_{\text {trap }}}\right)^{n},
$$

where $M_{\mathrm{Pl}} \simeq 2.4 \times 10^{18} \mathrm{GeV}$. Using the middle expression of (C2) with $m_{a}^{(0)} \simeq 0.25 T_{\mathrm{QCD}}^{2} / f_{a}$ to eliminate $T_{\text {trap }}$, this amounts to the following upper bound on $T_{\mathrm{LD}}$ :

$$
\begin{aligned}
T_{\mathrm{LD}} & \ll 6.8\left(\frac{\eta_{B} g_{* S}^{\text {trap }}}{c_{\mathrm{LD}}} T_{\mathrm{QCD}}\right)^{\frac{n+2}{2 n+4}}\left(\frac{M_{\mathrm{Pl}}}{\sqrt{g_{*}^{\text {trap }}}}\right)^{\frac{n+3}{2 n+4}}\left(\frac{1}{f_{a}}\right)^{\frac{1}{2 n+4}} \\
& \sim 3 \times 10^{2} \mathrm{TeV}\left(\frac{0.04}{c_{\mathrm{LD}}} \frac{g_{* S}^{\text {trap }}}{100}\right)^{\frac{1}{2}}\left(\frac{100}{g_{*}^{\text {trap }}}\right)^{\frac{7}{24}}\left(\frac{10^{9} \mathrm{GeV}}{f_{a}}\right)^{\frac{1}{12}} .
\end{aligned}
$$


[1] N. Arkani-Hamed and Y. Grossman, Light active and sterile neutrinos from compositeness, Phys. Lett. B 459, 179 (1999).

[2] T. Gherghetta, Dirac Neutrino Masses with Planck Scale Lepton Number Violation, Phys. Rev. Lett. 92, 161601 (2004).

[3] R. T. Co and K. Harigaya, Axiogenesis, Phys. Rev. Lett. 124, 111602 (2020).

[4] R. T. Co, L. J. Hall, and K. Harigaya, Axion Kinetic Misalignment Mechanism, Phys. Rev. Lett. 124, 251802 (2020).

[5] R. T. Co, L. J. Hall, and K. Harigaya, Predictions for axion couplings from ALP cogenesis, J. High Energy Phys. 01 (2021) 172.

[6] S. Dimopoulos, S. Raby, and L. Susskind, Light composite fermions, Nucl. Phys. B173, 208 (1980).

[7] C. Csáki, H. Murayama, and O. Telem, Some exact results in chiral gauge theories, Phys. Rev. D 104, 065018 (2021).

[8] P. B. Smith, A. Karasik, N. Lohitsiri, and D. Tong, On discrete anomalies in chiral gauge theories, arXiv: 2106.06402.

[9] M. D'Onofrio, K. Rummukainen, and A. Tranberg, Sphaleron Rate in the Minimal Standard Model, Phys. Rev. Lett. 113, 141602 (2014).

[10] R. T. Co, N. Fernandez, A. Ghalsasi, L. J. Hall, and K. Harigaya, Lepto-axiogenesis, J. High Energy Phys. 03 (2021) 017.

[11] K. Harigaya and R. Wang, Axiogenesis from $S U(2)_{R}$ phase transition, J. High Energy Phys. 10 (2021) 022.

[12] J. Preskill, M. B. Wise, and F. Wilczek, Cosmology of the invisible axion, Phys. Lett. 120B, 127 (1983).

[13] L. F. Abbott and P. Sikivie, A cosmological bound on the invisible axion, Phys. Lett. 120B, 133 (1983).

[14] M. Dine and W. Fischler, The not so harmless axion, Phys. Lett. 120B, 137 (1983).

[15] S. Borsanyi et al., Calculation of the axion mass based on high-temperature lattice quantum chromodynamics, Nature (London) 539, 69 (2016).

[16] S. Hannestad, A. Mirizzi, G. G. Raffelt, and Y. Y. Y. Wong, Neutrino and axion hot dark matter bounds after WMAP-7, J. Cosmol. Astropart. Phys. 08 (2010) 001.

[17] J. H. Chang, R. Essig, and S. D. McDermott, Supernova 1987A constraints on sub-GeV dark sectors, millicharged particles, the QCD axion, and an axion-like particle, J. High Energy Phys. 09 (2018) 051.

[18] P. Carenza, T. Fischer, M. Giannotti, G. Guo, G. MartínezPinedo, and A. Mirizzi, Improved axion emissivity from a supernova via nucleon-nucleon bremsstrahlung, J. Cosmol.
Astropart. Phys. 10 (2019) 016; Erratum, J. Cosmol. Astropart. Phys. 05 (2020) E01.

[19] A. Ayala, I. Domínguez, M. Giannotti, A. Mirizzi, and O. Straniero, Revisiting the Bound on Axion-Photon Coupling from Globular Clusters, Phys. Rev. Lett. 113, 191302 (2014).

[20] K. Hamaguchi, N. Nagata, K. Yanagi, and J. Zheng, Limit on the axion decay constant from the cooling neutron star in cassiopeia A, Phys. Rev. D 98, 103015 (2018).

[21] T. Braine et al. (ADMX Collaboration), Extended Search for the Invisible Axion with the Axion Dark Matter Experiment, Phys. Rev. Lett. 124, 101303 (2020).

[22] B. T. McAllister, G. Flower, E. N. Ivanov, M. Goryachev, J. Bourhill, and M.E. Tobar, The ORGAN experiment: An axion haloscope above $15 \mathrm{GHz}$, Phys. Dark Universe 18, 67 (2017).

[23] A. A. Melcón et al., Axion searches with microwave filters: The RADES project, J. Cosmol. Astropart. Phys. 05 (2018) 040.

[24] W. Chung, CULTASK, the coldest axion experiment at CAPP/IBS in Korea, Proc. Sci., CORFU2015 (2016) 047.

[25] D. Alesini, D. Babusci, D. Di Gioacchino, C. Gatti, G. Lamanna, and C. Ligi, The KLASH proposal, arXiv: 1707.06010 .

[26] A. Caldwell, G. Dvali, B. Majorovits, A. Millar, G. Raffelt, J. Redondo, O. Reimann, F. Simon, and F. Steffen (MADMAX Working Group Collaboration), Dielectric haloscopes: A new way to detect axion dark matter, Phys. Rev. Lett. 118, 091801 (2017).

[27] P. Brun et al. (MADMAX Collaboration), A new experimental approach to probe QCD axion dark matter in the mass range above $40 \mu \mathrm{eV}$, Eur. Phys. J. C 79, 186 (2019).

[28] A. Arvanitaki, S. Dimopoulos, and K. Van Tilburg, Resonant Absorption of Bosonic Dark Matter in Molecules, Phys. Rev. X 8, 041001 (2018).

[29] E. Armengaud et al. (IAXO Collaboration), Physics potential of the International Axion Observatory (IAXO), J. Cosmol. Astropart. Phys. 06 (2019) 047.

[30] N. Aghanim et al. (Planck Collaboration), Planck 2018 results. VI. Cosmological parameters, Astron. Astrophys. 641, A6 (2020).

[31] K. N. Abazajian et al. (CMB-S4 Collaboration), CMB-S4 Science Book, First Edition, arXiv:1610.02743.

[32] J. Delabrouille et al. (CORE Collaboration), Exploring cosmic origins with CORE: Survey requirements and mission design, J. Cosmol. Astropart. Phys. 04 (2018) 014.

[33] V. Domcke, Y. Ema, K. Mukaida, and M. Yamada, Spontaneous baryogenesis from axions with generic couplings, J. High Energy Phys. 08 (2020) 096. 